Graphique 1 • Pourcentage d'enseignants indiquant avoir dans leur classe plus de 10 \% d'élèves présentant des problèmes de comportement

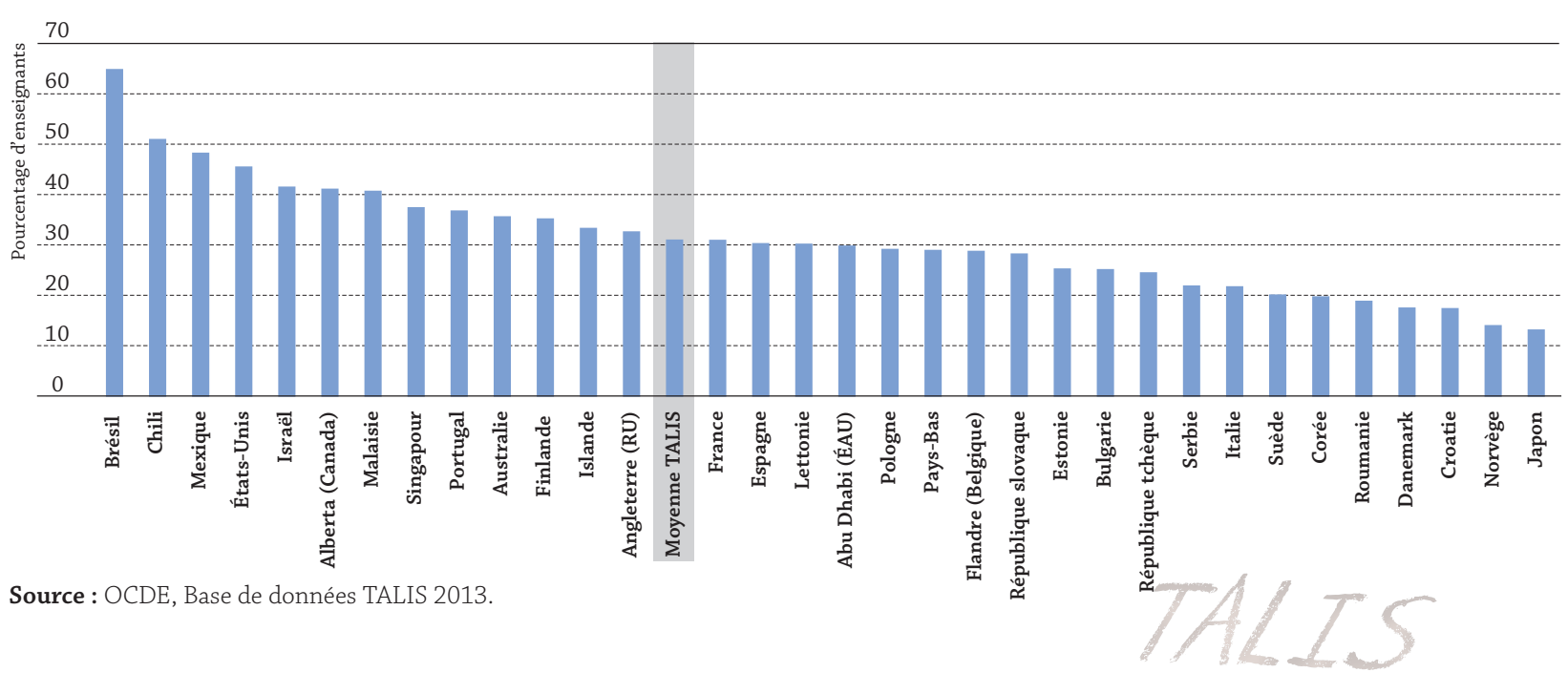

Dans l'ensemble des pays et économies ayant participé à l'enquête TALIS 2013, les enseignants indiquant avoir dans leur classe des pourcentages plus importants d'élèves présentant des problèmes de comportement sont également ceux qui déclarent consacrer davantage de temps au maintien de l'ordre en classe (en moyenne, $19 \%$ de leur temps de classe, contre $10 \%$ pour les pays où le pourcentage de ce type d'élèves est plus faible ; voir le graphique 2). Ainsi, plus les classes sont difficiles, plus la part du temps d'enseignement qui doit être consacrée au maintien de l'ordre est importante - dans une mesure proche du double, en moyenne.
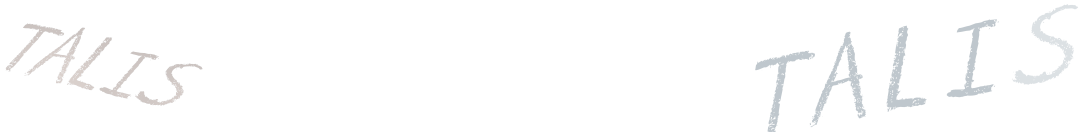

Graphique 2 • Pourcentage moyen du temps de classe consacré au maintien de l'ordre, selon le pourcentage d'élèves ayant des problèmes de comportement

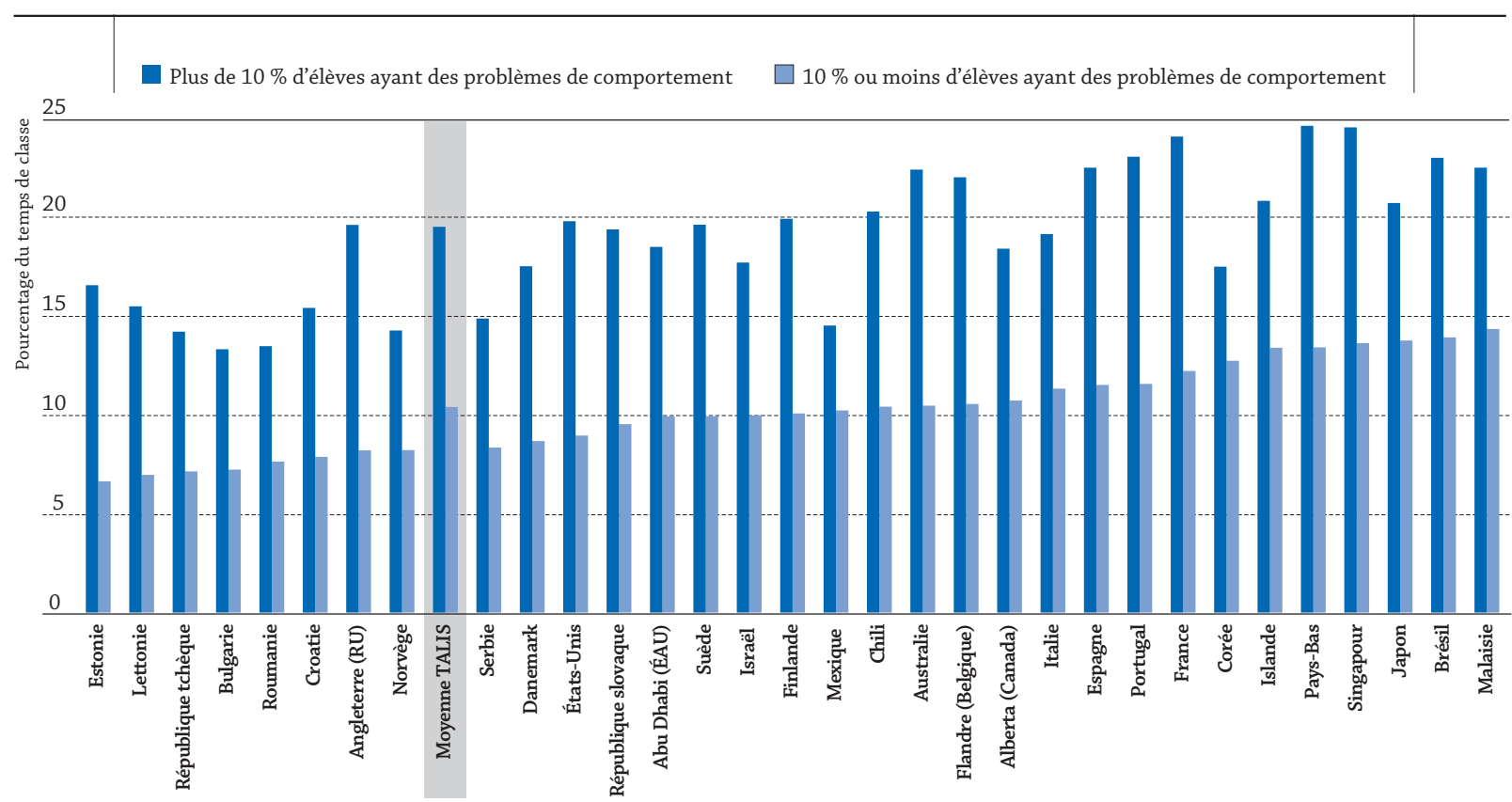

Source : OCDE, Base de données TALIS 2013. 


\section{Absentéisme des élèves}

Outre le temps perdu en classe lorsque les enseignants doivent se consacrer au maintien de l'ordre au lieu de dispenser leur enseignement, les élèves passent également à côté de possibilités d'apprentissage lorsqu'ils manquent régulièrement des cours.

Dans $\mathrm{x}$ les pays et économies ayant participé à l'enquête TALIS, 39 \% des enseignants travaillent dans des établissements où des élèves sont absents chaque semaine. En Alberta (Canada), aux États-Unis, en Finlande et en Suède, toutefois, ce problème est encore plus prononcé, plus de 60 \% des enseignants travaillant dans des établissements dont le chef d'établissement indique un absentéisme hebdomadaire de la part des élèves.

\section{Qu'est-ce que cela signifie dans la pratique?}

Les élèves peuvent manquer l'école pour de multiples raisons, notamment médicales ou familiales. Mais certains ne vont pas en cours ou ont un comportement perturbateur simplement parce qu'ils ne voient pas l'intérêt d'être à l'école. Les établissements où les taux d'absentéisme sont particulièrement élevés doivent s'efforcer de motiver leurs élèves. Lorsqu'on leur propose un travail intéressant dont le niveau de difficulté est optimal (c'est-à-dire ni trop faible, ni trop élevé), les élèves croient en leur capacité à faire ce qui est demandé et sont plus motivés pour suivre les cours et y participer (Porter, 2006 ; Willms et al., 2009).

\section{Intimidation et agression verbale parmi les élèves}

Les actes d'intimidation ou d'agression verbale parmi les élèves peuvent constituer un autre obstacle à l'apprentissage. Dans les pays et économies ayant participé à l'enquête TALIS, 16 \% des enseignants travaillent dans des établissements dont le chef d'établissement indique que des actes de ce type se produisent au moins chaque semaine. Toutefois, en Alberta (Canada), en Australie, au Brésil, en Finlande, en Flandre (Belgique), au Mexique et en Suède, c'est au moins un enseignant sur quatre qui est dans ce cas.

Parmi les pays et économies ayant participé à l'enquête TALIS, ceux qui font état d'une plus grande prévalence des actes d'intimidation ou d'agression verbale parmi les élèves sont également plus susceptibles d'indiquer des taux d'absentéisme plus élevés chez ces derniers (voir le graphique 3). Ces aspects négatifs peuvent ainsi s'aggraver mutuellement et créer un environnement d'apprentissage négatif.

\section{Graphique 3 - Relation entre la prévalence des actes d'intimidation ou d'agression verbale} parmi les élèves et l'absentéisme de ces derniers ${ }^{1}$

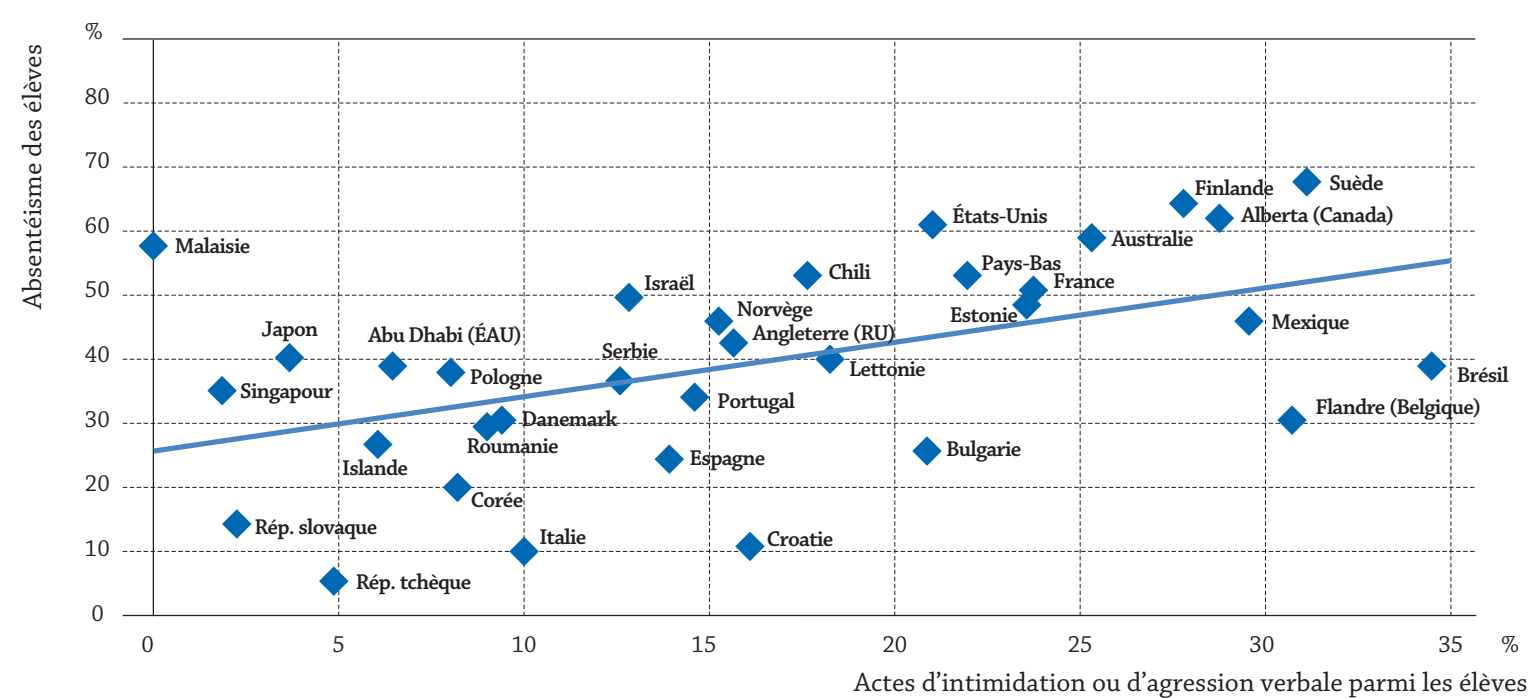

1. Coefficient de corrélation de Pearson $\mathrm{r}=0.50$.

Source : OCDE, Base de données TALIS 2013. 


\section{Climat de l'établissement et temps consacré à l'apprentissage}

Créer une culture positive au sein de l'établissement peut être bénéfique pour l'apprentissage. Pour ce faire, il est par exemple possible d'impliquer les élèves, les parents et les enseignants dans les décisions concernant l'établissement. De fait, dans les pays et économies ayant participé à l'enquête TALIS, les enseignants qui déclarent travailler dans un établissement où le niveau de participation parmi les parties prenantes* est plus élevé sont moins susceptibles de faire état de forts pourcentages d'élèves ayant des problèmes de comportement dans leur classe (voir le graphique 4). Ces résultats montrent qu'en encourageant la participation des parties prenantes dans les décisions concernant l'établissement, tout en favorisant une culture de partage des responsabilités et de soutien mutuel, il est possible d'améliorer le comportement des élèves et l'environnement d'apprentissage.

\section{Graphique 4 • Relation entre le niveau déclaré de participation des parties prenantes et le pourcentage d'élèves ayant des problèmes de comportement en classe}

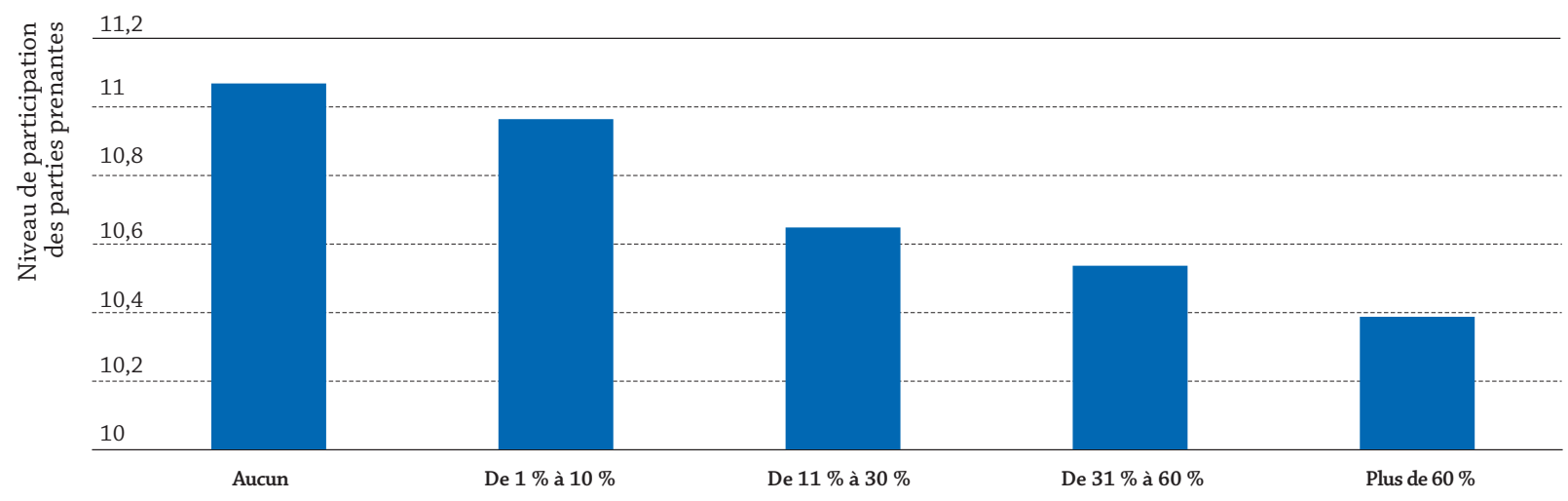

Source : OCDE, Base de données TALIS 2013

Pourcentage d'élèves ayant des problèmes de comportement

Pour conclure Le comportement perturbateur des élèves réduit le temps consacré à l'enseignement et donc par là même les possibilités d'apprentissage de tous les élèves d'une classe, en plus d'affecter l'assiduité des élèves en cours. Les établissements peuvent contribuer à atténuer ces difficultés en encourageant les initiatives permettant l'établissement de relations positives parmi les élèves et entre ces derniers, leurs parents et leurs enseignants. Ainsi, l'implication de l'ensemble de ces parties prenantes dans les décisions concernant l'établissement, et la mise en place ou le renforcement d'une culture de partage des responsabilités, sont susceptibles d’améliorer la motivation des élèves et de les amener à se comporter de façon plus positive (Porter, 2006).

\section{Références}

Porter, L. (2006), Behaviour in Schools: Theory and Practice for Teachers, Open University Press, Maidenhead.

Willms, J., S. Friesen et P. Milton (2009), Qu'as-tu fait à l'école aujourd'hui ? Transformer les salles de classe par l'engagement social, scolaire et intellectuel, premier rapport national, Association canadienne d'éducation, Toronto.

* L'indice TALIS de participation des parties prenantes est tiré de questions sur le degré de participation des élèves, des parents et du personnel aux décisions concernant l'établissement, et sur l'existence ou non au sein de l'établissement d'une culture de partage des responsabilités et de soutien mutuel. Les valeurs de cet indice sont comprises entre 4.11 et 15.77 .

\section{Voir}

www.oecd.org/talis

Les indicateurs de l'éducation

à la loupe

PISA à la loupe

Contacter
Gabriela Miranda Moriconi
(gmoriconi@fcc.org.br)
Katarzyna Kubacka
(katarzyna.kubacka@oecd.org)

Pour en savoir plus

OCDE (2014), Résultats de TALIS 2013 : Une perspective internationale sur l'enseignement et l'apprentissage, TALIS, Éditions OCDE, Paris.

OCDE (2014), Guide TALIS 2013 à l'intention des enseignants, TALIS, Éditions OCDE, Paris.

Ce document est publié sous la responsabilité du Secrétaire général de l'OCDE. Les opinions qui y sont exprimées et les arguments qui y sont employés ne reflètent pas nécessairement les vues officielles des pays membres de l'OCDE

Ce document et toute carte qu'il peut comprendre sont sans préjudice du statut de tout territoire, de la souveraineté s'exerçant sur ce dernier, du tracé des frontières et limites internationales, et du nom de tout territoire, ville ou région.

Les données statistiques concernant Israël sont fournies par et sous la responsabilité des autorités israéliennes compétentes. L'utilisation de ces données par l'OCDE est sans préjudice du statut des hauteurs du Golan, de Jérusalem-Est et des colonies de peuplement israéliennes en Cisjordanie aux termes du droit international.

Vous êtes autorisés à copier, télécharger ou imprimer du contenu OCDE pour votre utilisation personnelle. Vous pouvez inclure des extraits des publications, des bases de données et produits multimédia de l'OCDE dans vos documents, présentations, blogs, sites Internet et matériel d'enseignement, sous réserve de faire mention de la source OCDE et du copyright. Les demandes pour usage public ou commercial ou de traduction devront être adressées à rights@oecd.org. ๑) Fuse/Getty Images @ Image Source/Getty Images @ Laurence Mouton/PhotoAlto Agency RF Collections/Inmagine ltb. 